\title{
Buckling Behavior Monitoring of Composite Wing Box Model Using Fiber Bragg Grating Sensor System
}

\author{
Chang-Sun Hong ${ }^{*}$, Chi-Young Ryu, Jung-Ryul Lee, and Chun-Gon Kim \\ Department of Mechanical Engineering \\ Division of Aerospace Engineering \\ Korea Advanced Institute of Science and Technology (KAIST), \\ 373-1, Kusong-dong, Yusong-gu, Taejon, 305-338, Korea
}

\begin{abstract}
Advanced composites are being extensively used for aerospace structures due to the high stiffness to weight and high strength to weight ratios. Measuring internal strains of composite structures is of great interest with respect to the structural integrity of aerospace structures. A large number of sensors are required for large-scale structures such as aircraft. Fiber Bragg grating (FBG) sensor system based on the wavelength division multiplexing (WDM) technology offers a versatile and powerful one for strain monitoring of large structures due to the advantage of multiplexing capability.

In this paper, we present an improved FBG sensor system using a wavelength-swept fiber laser (WSFL). The WSFL provides unique and powerful output characteristics useful for a large number of sensor interrogations without any other expensive optic devices such as optical switches. As a practical application of aircraft structures, we demonstrate 24 FBG sensors were used to monitor strains of the smart composite wing box model in the bending test. 3 sensor lines are embedded into upper skin and 1 sensor line is embedded into front spar of composite wing box. Each sensor line has 6 FBG for the strain sensing and 1 reference FBG for temperature compensation. Experimental results are compared with finite element analytical results. The structural bending behavior of composite wing box monitored by FBG sensors shows an almost same tendency with the analytical result. All strain data can be real-timely visualized and saved in PC.
\end{abstract}

Keywords: Fiber Bragg grating sensor system, wavelength-swept fiber laser, real-time signal-processing, strain monitoring, composite wing box, buckling behavior monitoring

\section{INTRODUCTION}

Most of the conventional damage-assessment and nondestructive inspection methods are time-consuming and are often difficult to implement on hard-to-reach-parts of the structure. For these reasons, built-in assessment system must be developed to monitor constantly the structural integrity of critical components. Measuring structural response in the form of strains and deflections is of great interest. Optical fiber sensors (OFS) have shown a potential to serve real time health monitoring of the structures. They can be easily embedded or attached to the structures and are not affected by the electromagnetic field. Also, they have the flexibility of the sensor size $(\mu \mathrm{m} \sim \mathrm{km})$ and very highly sensitive. These advantages of OFS make it to be the potential solution for sensor systems of smart structures ${ }^{1}$. Recently, fiber optic sensors have been introduced into composite structures ${ }^{2 \sim 4}$. Advanced composites are being extensively used for aerospace structures due to the high stiffness to weight and high strength to weight ratios. The stiffened wing boxes made of composite materials can be applied to aircraft fuselage or wing structure. The advantage of a composite wing box is that it satisfies the requirements of increased stiffness and reduced weight. Therefore, it is important to investigate the structural behavior of a composite wing box. Measuring internal strains of composite structures is of great interest with respect to the structural integrity of aerospace structures. FBG sensor based on the WDM technology attracts considerable research interest and appears to be ideally suited for structural health monitoring of composite structures. FBG sensor is easy to be multiplexed and has many advantages of linear response, absolute measurement, etc. As the spectral signature renders the measurement free from intensity fluctuations, it guarantees reproducible measurements despite optical losses due to bending and/or connectors.

\footnotetext{
Correspondence: Email: cshong@ kaist.ac.kr; WWW: http://smartech.kaist.ac.kr; Telephone: 82-42-869-3712;
} 
Proper monitoring of measurands in FBG sensor systems requires accurate measurement of the Bragg center wavelength, and the ability to track rapid shifts of the wavelength especially in the structures under dynamic loading. Various interrogation schemes have been reported for the detection of small Bragg wavelength shifts based on the combination of a broadband source and a wavelength-dependent receiver. LED's, amplified spontaneous emission sources and ultrashortpulse lasers are typically used as broadband sources. For wavelength-dependent receivers, scanning tunable filters ${ }^{5}$ and unbalanced interferometers ${ }^{6}$ have been employed. However, these schemes have shown some drawbacks associated with low signal powers by using a narrow spectral slice from a broad source spectrum. Moreover, these results showed poor spectral resolution determined by the resolution of the tunable filter or the spectrometer itself. Recently, the interrogation technique based on the WSFL was developed ${ }^{7}$. This technique offers several attractive features. First, it provides for high signal powers, since the full source output is available during the measurement of a given grating's Bragg wavelength. Second, the broad source tuning range and narrow instantaneous spectral line width allow for a large number of individual elements within the array. The FBG sensor system using WSFL was constructed in the previous study, and performance of the system was also verified ${ }^{8}$.

In this study, the behavior of composite wing box embedded 24 FBG sensors was real-timely monitored in the bending test. We constructed an improved FBG sensor system using a WSFL and a signal-processing board with an electrical circuit for the test. In order to monitor the structural strain real-timely, the signal-processing program was also constructed using Labview software for storage and visualizing of the data. Experiments showed that the constructed FBG sensor system and the real-time signal-processing program could successfully monitor the behavior of composite wing box. It was successfully demonstrated that the constructed FBG sensor system could be useful for the large structures that require a large number of sensor arrays.

\section{FBG SENSOR SYSTEM}

\subsection{Construction of a WSFL and sensor arrays with reference sensors}

The accurate detection of Bragg wavelength shift is important to strain measurement. For the purpose, the WSFL is constructed and employed to the present FBG sensor system. The WSFL has a scanning tunable filter in the cavity to sweep the laser output wavelength in time continuously and repeatedly over a range of a few tens of nanometers. When the WSFL output is directed into the grating array, the reflected optical signal consists of a series of pulses in the time domain whose timing relative to the start of the wavelength sweep is determined by both the Bragg wavelength of each corresponding grating and the position of each grating within the array. By measuring the reflected pulse timing characteristics and employing simple signal processing schemes based, for example, on time interval counting ${ }^{8}$ or peak detection as in this study, one can deduce the instantaneous Bragg wavelength of the individual gratings within the array.

Fig. 1(a) shows a schematic of the configuration of the WSFL and (b) the grating arrays with reference FBGs and an F-P etalon. The WSFL was in a unidirectional ring configuration with isolators, a 3-dB output coupler, and an $\mathrm{Er}^{3+}$-doped fiber pumped by a laser diode at $980 \mathrm{~nm}$. An F-P tunable filter was used as the intracavity scanning filter and had a 3-dB bandwidth of $0.27 \mathrm{~nm}$ and a free spectral range of $58 \mathrm{~nm}$. We modulate the F-P filter with a triangular waveform to produce a wavelength sweep over $40 \mathrm{~nm}$ from 1525 to $1565 \mathrm{~nm}$ at a $200 \mathrm{~Hz}$ repetition rate. The laser output was directed into four arrays of sensing gratings and reference gratings for temperature compensation. It was possible to expand sensor array to four lines using additional couplers and detectors aided by high signal output power of WSFL. So this sensor system has more advantage than the system that uses expensive optic devices such as optical switches ${ }^{9}$. The average output power of the laser was $2.22 \mathrm{~mW}$ at a pump power of $43 \mathrm{~mW}$, with a variation of less than $1 \mathrm{~dB}$ across the full wavelength sweep. The output power of the WSFL was over 1000 times as large as that of amplified spontaneous emission (ASE) of an LD-pumped $\mathrm{Er}^{3+}$-doped fiber (EDF) as usually used for the light source in the FBG sensor system.

An F-P etalon whose reflected signal has $1 \mathrm{~nm}$ free spectral range (FSR), the spacing between each resonance, was fabricated in order to remove the wavelength non-linearity of the WSFL output. Since the general PZT has hysteresis, the PZT of an F-P tunable filter may be non-linear with respect to a linear modulation signal. Errors caused by this phenomenon can be corrected using an F-P etalon. The multi-beam interference optical signal of the F-P etalon was used for a wavelength grid line that have identical wavelength spacing. In this study, we manufactured the F-P etalon to have a FSR of $1 \mathrm{~nm}$. If an FBG of known Bragg center wavelength is located anywhere in the wavelength grid line, a coordinate of wavelength can be generated. The information from the reference FBG and the F-P etalon is used for processing the strain measurement. The WSFL with a reference FBG and the F-P etalon made for these purpose was named to WSFLWI (WSFL with wavelength indicators) in this study. As stated above, total 24 FBG sensors were embedded into the composite wing 
box. Each sensor line has 6 sensors and a reference FBG as shown in Fig. 1(b). The Bragg wavelength difference between neighboring sensors was about $4 \mathrm{~nm}$.

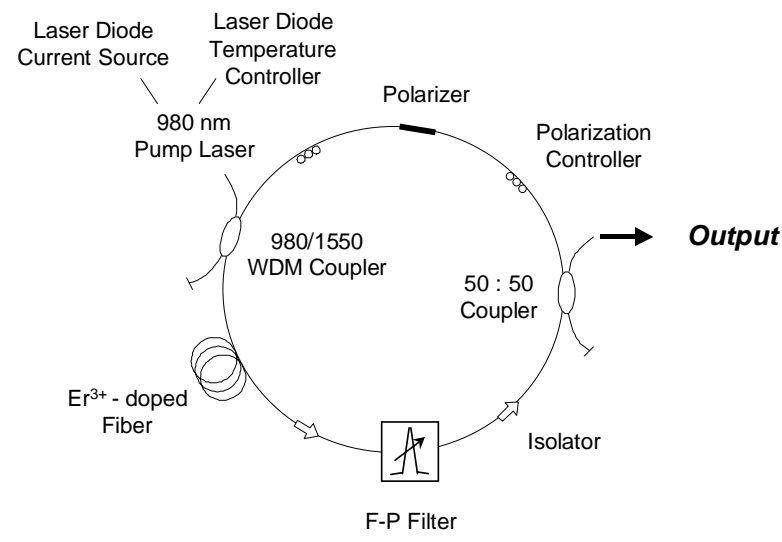

(a)

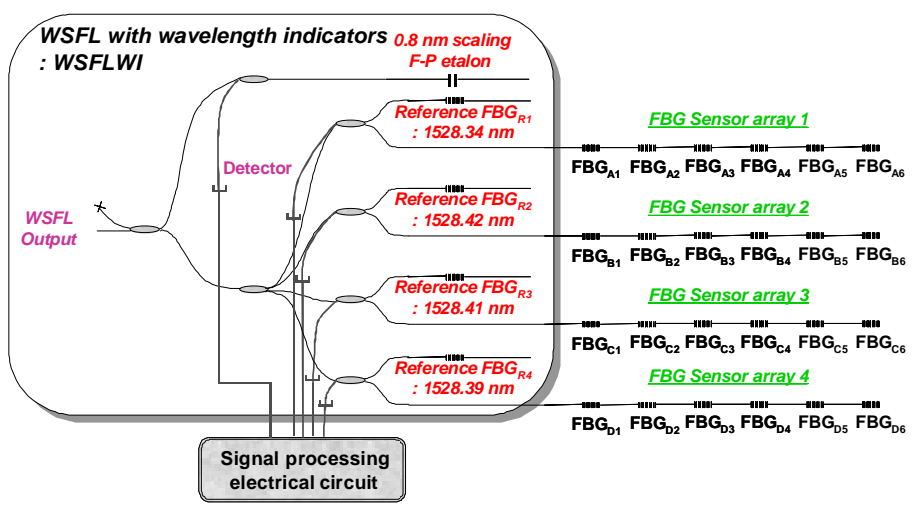

(b)

Fig. 1 Configuration of (a) the wavelength-swept fiber laser and (b) the grating sensor arrays.

\subsection{Real-time signal processing scheme}

As shown in Fig.1(b), the sensor signals of each sensor arrays are measured with photo detectors. These are wavelengthencoded signals in the time domain as shown in Fig. 2(a). The triangular waveform shown in Fig. 2 (a) is the $200 \mathrm{~Hz}$ electrical signal that was applied to the scanning filter. As the voltage of the signal increased, the output wavelength increased and vice versa.

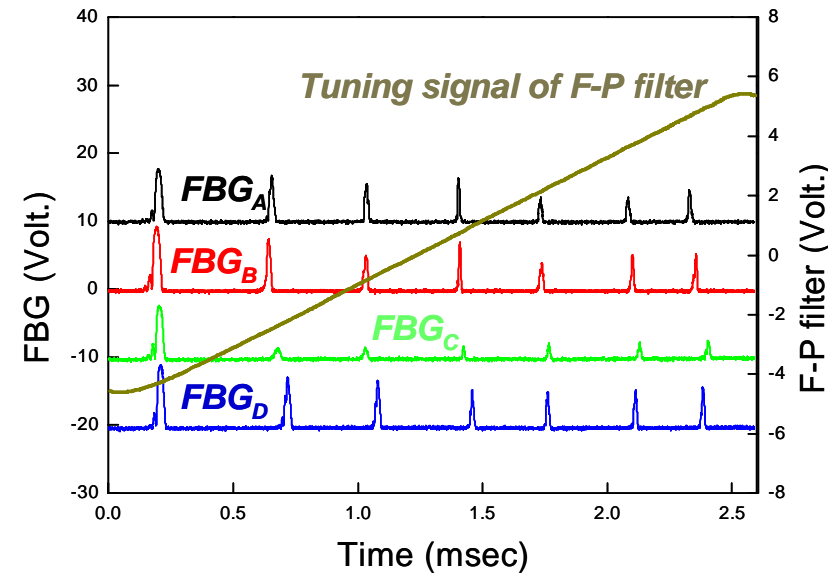

(a)

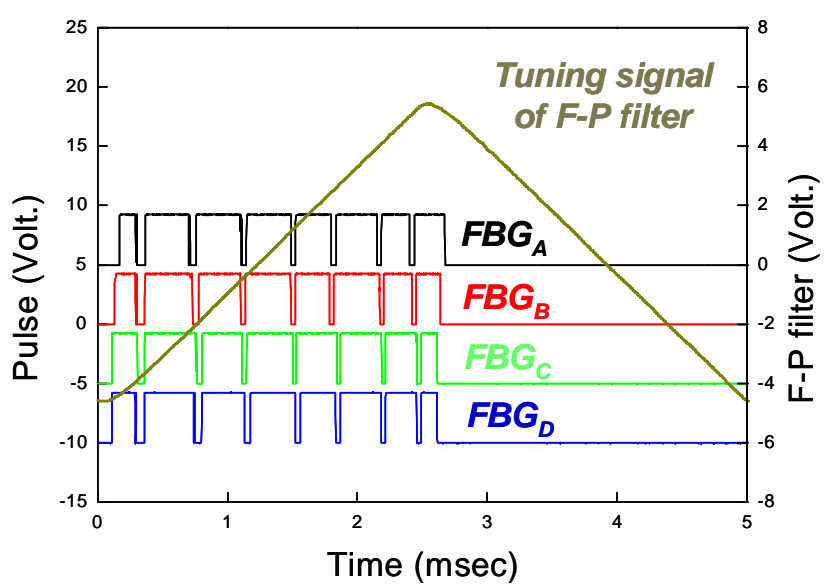

(b)

Fig. 2 (a) Original sensor signals, (b) Processed signals by electrical circuit.

Electrical signal-processing scheme was utilized to measure the wavelength shift of each sensor. The process is as follows. First, the analog voltage differentiation circuit and zero-crossing comparator execute peak detection of FBG sensors. Second, at the peak point of the sensors, the derivative signal crosses zero voltage and voltage comparator generates a digital pulse signal such as rising edge (from 0 to 5 Volt.) as shown in Fig. 2(b). Since these sensor signals are changed to digital signal, it is possible to count time-interval between rising edges, i.e. peaks of FBG sensors by high speed counter of $20 \mathrm{MHz}$ and the counted numbers are transferred to the personal computer. By measuring the counted number of each sensor from the reference sensor and matching the counted number of each sensor to grid counts of the F-P etalon with $1 \mathrm{~nm}$ interval, the wavelength-shift of each sensor can be calculated. When FBG sensors are in the same environmental temperature and calibrated by temperature compensating reference FBG sensor, the strain is calculated by the following simplified relation between strain and Bragg wavelength shift. 


$$
\varepsilon=\frac{1}{\left(1-p_{e}\right)} \frac{\Delta \lambda_{B}}{\lambda_{B}}
$$

where $p_{e}(=0.225)$ is the photo-elastic constant and measured experimentally ${ }^{10}$. Procedures of strain calculation were programmed by Labview software with GUI. The real-time strain monitoring window that displays the strain histories of the FBG sensor could be constructed by this software. The constructed FBG sensor system showed a good strain resolution less than $10 \mu \varepsilon$.

\section{FABRICATION AND TESTING OF A COMPOSITE WING BOX}

The cocuring of a stiffened composite plate and a stiffened wing box was fabricated by the autoclave. The geometry and stacking sequence of composite wing box is shown in Fig. 3. It was composed of two spars, four stiffeners, and two skins. Both the ends of the wing box were designed for clamping. Both the spars and the skins had the same stacking sequences, $\left[0_{2} / 90 / \pm 45\right]_{S}$, and stiffeners had the stacking sequence of $\left[00_{2} / 90 / 45 / 0 /-45\right]_{S}$.

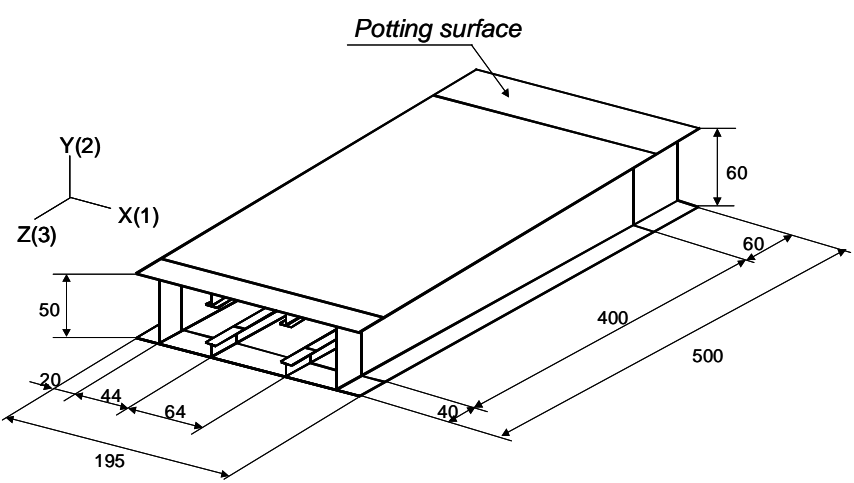

(a)

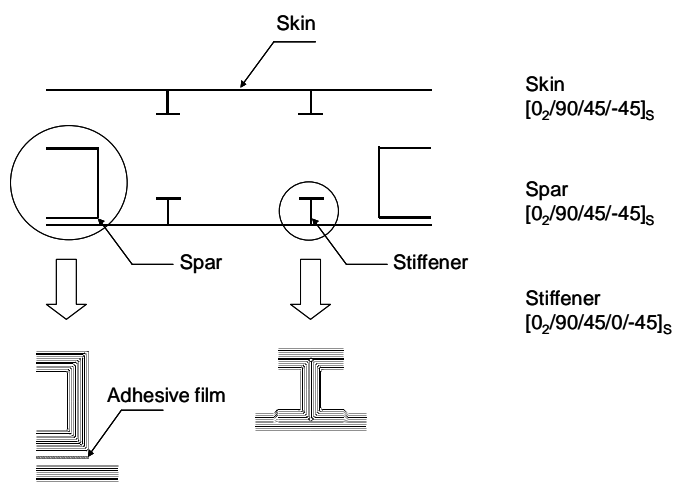

(b)

Fig. 3 (a) Geometry, (b) Stacking sequence of composite win box.

The cross section of the wing consists of multi-cell box beam. The components for the upper skin, the lower skin, the front spar, the rear spar and the stiffeners were assembled for each part. The one method of the fabrication of the wing box is secondary bonding of all components. The other method is the using of both cocuring and secondary bonding. The latter was used in this paper. Cocuring was done for two parts. The upper skin, and two I-stiffeners assembled the one part. The lower skin and two I-stiffeners assembled the other part. These two cocured stiffened skins were then secondary bonded by FM-73M adhesive film with two spars. The clamping jig was used for bonding of two parts. The assembled parts were then cured according to the curing cycle of the adhesive film. Before bonding, the surface to be bonded was polished by the \#150 sandpaper. The composite wing box was fabricated using graphite/epoxy prepreg. Graphite/Epoxy perpreg was used for the stiffened composite plate and the composite wing box. The ply thickness was $0.125 \mathrm{~mm}$. The material properties were $\mathrm{E}_{1}=$ $130.0 \mathrm{GPa}, \mathrm{E}_{2}=\mathrm{E}_{3}=10.0 \mathrm{GPa}, \mathrm{G}_{12}=\mathrm{G}_{13}=4.85 \mathrm{GPa}, \mathrm{G}_{23}=3.62 \mathrm{GPa}, v_{12}=v_{13}=0.31, v_{23}=0.52, \mathrm{X}_{\mathrm{T}}=1933 \mathrm{MPa}, \mathrm{X}_{\mathrm{C}}=1051$ $\mathrm{MPa}, \mathrm{Y}_{\mathrm{T}}=51 \mathrm{MPa}, \mathrm{Y}_{\mathrm{C}}=141 \mathrm{MPa}, \mathrm{S}=61 \mathrm{MPa}$ where $\mathrm{X}$ is the strength in the fiber direction, $\mathrm{Y}$ is the strength in transverse direction, subscripts $\mathrm{T}$ and $\mathrm{C}$ represent tension and compression, respectively.

I-stiffeners were formed by the continuous lay-up of the skin to improve the integrity. Three $0^{\circ}$ plies of the flange were continuously laid up into the skin. Unidirectional tape fillers were inserted into the junction parts of stiffeners. The curing cycle was the standard cycle for graphite/epoxy composite materials. As previously stated, total 24 sensors were embedded in composite wing box. $\mathrm{FBG}_{\mathrm{A}}, \mathrm{FBG}_{\mathrm{B}}$, and $\mathrm{FBG}_{\mathrm{D}}$ lines were embedded in the upper skin. And $\mathrm{FBG}_{\mathrm{C}}$ line was embedded in the front spar. Fig. 4 shows notation and location of embedded FBG sensors. In order to monitor effectively the buckling behavior of composite wing box, the sensors of $\mathrm{FBG}_{\mathrm{A}}, \mathrm{FBG}_{\mathrm{B}}$, and $\mathrm{FBG}_{\mathrm{C}}$ lines were embedded symmetrically in laminated composites as like $[0 /\{0\} / 0 / 90 / \pm 45]_{S}$ where " \{\} " means the direction of embedded FBG sensors. The sensors of FBG line were embedded as like $\left[0 /\{0\} / 0 / 90 / 45 /-45 /-45 / 45 / 90 / 0_{2}\right]_{T}$ to monitor global behavior of composite wing box. In 
the Fig. 4, the subscript number 1, 2, and 3 means that FBG sensors were embedded into the outside of laminate composites, and the subscript number 4, 5, and 6 means that FBG sensors were embedded into the inside of laminate composites. The selection of location of sensors was determined using the analytic results by the general-purpose code, ABAQUS. The local buckling points where strain change is severe were selected as the sensing points under bending load. For example, the location of sensors in $\mathrm{FBG}_{\mathrm{A}}$ line was determined by referring to the analytic results as shown in Fig. 5. The location of sensors of the other lines was selected through the same procedures.
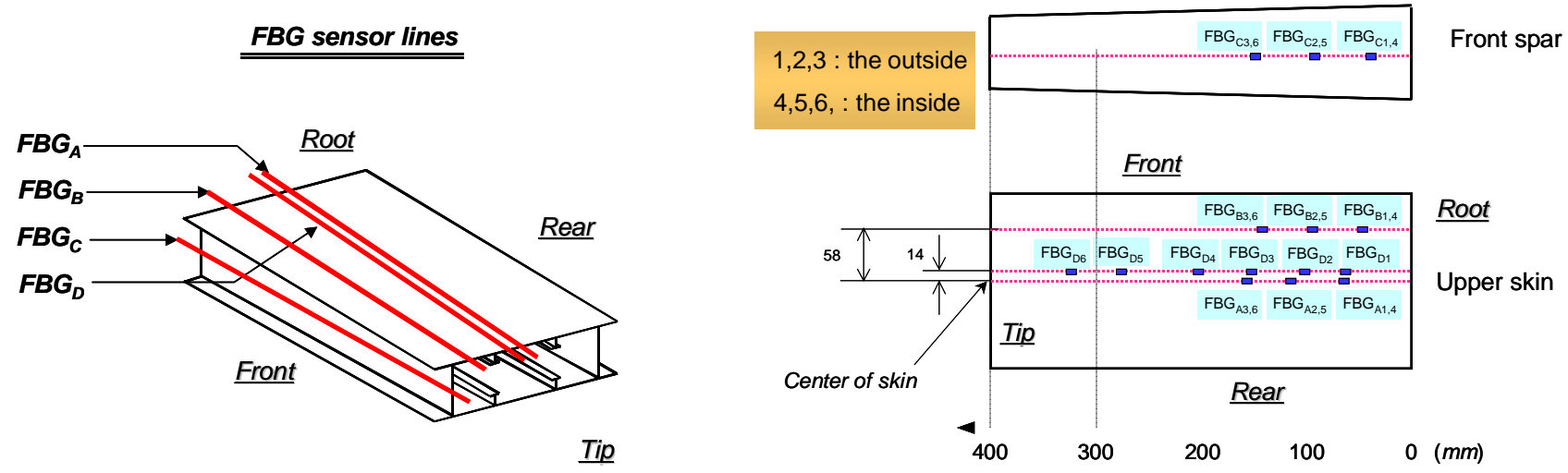

Fig. 4 Location and notation of FBG sensors.
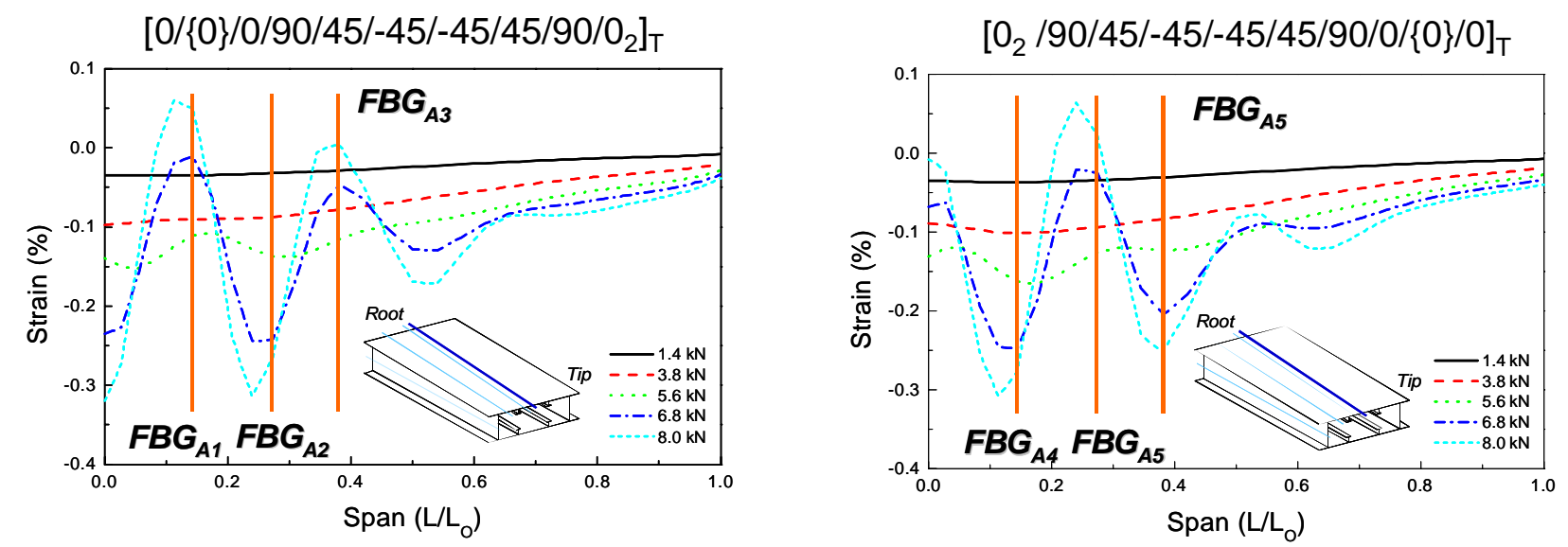

Fig. 5 Strains of upper skin along the center line under bending loading.
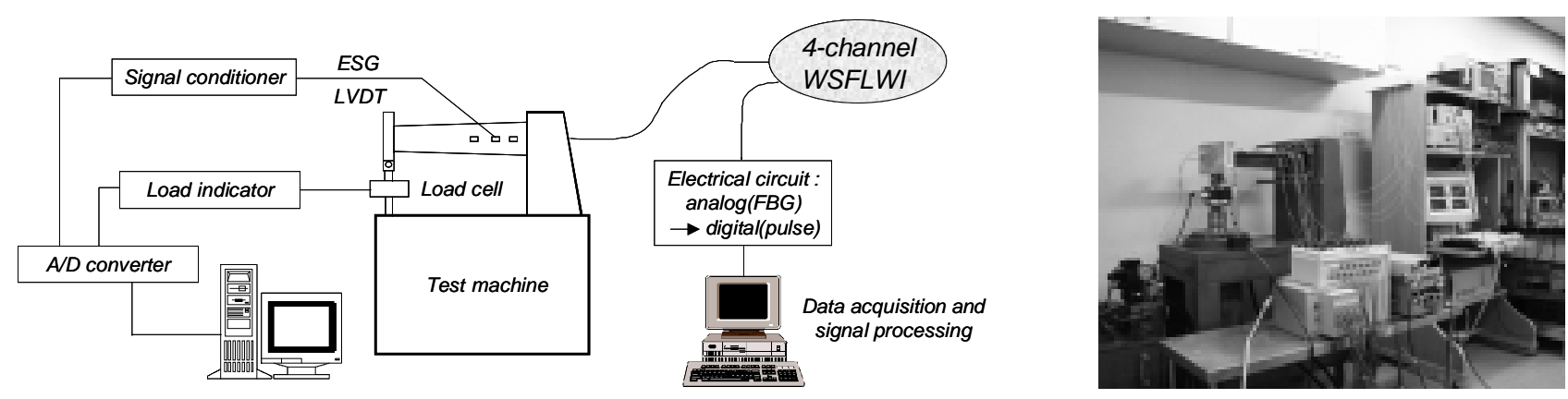

Fig. 6 Schematic and overall view of experimental setup for bending test of wing box structure. 
The both ends of the composite wing box were potted in the casting resin to add the role of rib. The schematic and overall view of experimental setup for bending test of composite wing box is shown in Fig. 6. The load cell was attached to the loading end. Electrical strain gages (ESG) were bonded on the surface of wing box in order to compare the measured strain with one by FBG sensors. A linear variable differential transformer (LVDT) was used to measure vertical displacement. The velocity of the loading was $1 \mathrm{~mm} / \mathrm{min}$. All strains from the FBG sensors were saved and visualized in real-time during the test.

\section{EXPERIMENTAL RESULTS}

Strain vs. time curves by FBG sensors were shown in Fig. 6. In the sensor line B and C, pop-in (knee-point) phenomena were definitely observed at $920 \mathrm{sec}$. These were caused by transient strain-release due to the failure of spar that plays a roll of bearing the most bending load. For the sensor line A and D that were relatively far apart from the spar, strain-release effect from the spar was weakly propagated. As bending load increased, compressive strain was increased in the upper skin. It was observed that the buckling behavior was deepened at the last stage of test for the sensor line B which was embedded in the upper skin between stiffener and spar. The deepening phenomena of buckling behavior were firstly occurred at the sensing point of 1, 4 closer to the clamped root of wing box. As bending load increased, these phenomena occurred at the sensing point of 2, 5 relatively apart from the root of wing box. It was obvious that the deepening poi nt of buckling behavior was progressed from the clamped root to the loading tip of composite wing box as shown in Fig. 6(b). These phenomena were also observed in the spar, as shown in Fig. 6(c).

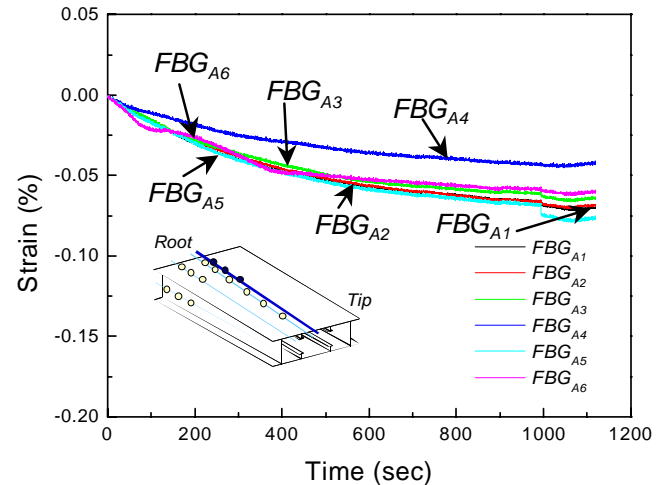

(a) Sensor line A

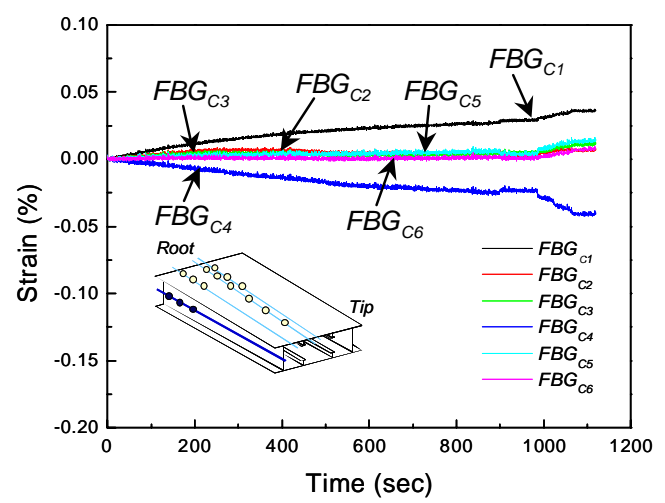

(c) Sensor line C

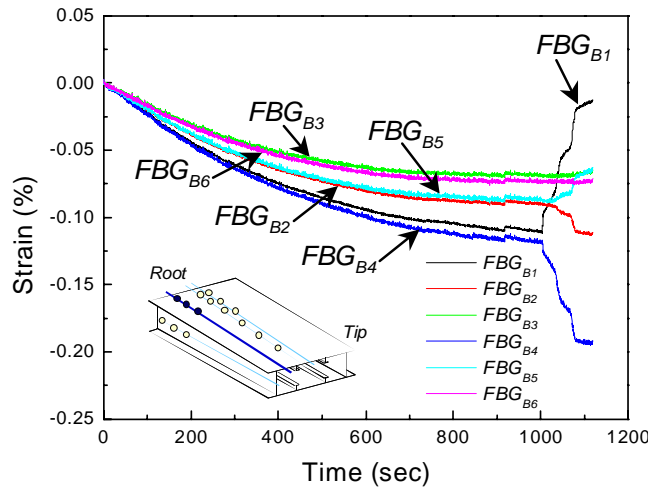

(b) Sensor line B

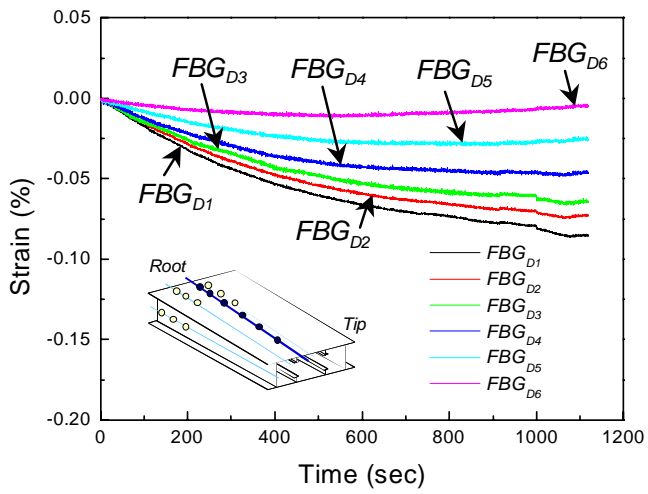

(d) Sensor line D

Fig. 6 Strain vs. time curve by FBG sensors during bending test of composite wing box.

The measured strain by FBG sensors was compared with strain by ESG and analytical results. In this paper, we analyzed the bending and buckling behavior of the composite wing box with the general-purpose code, ABAQUS. The stiffened composite plate of wing box was modeled using eight-noded, two-dimensional shell element, known as S8R5 element in ABAQUS. Multi-Point Constraints (MPCs) in ABAQUS were applied to the loaded edges. The loaded ends were uniformly 
shortened using MPCs. Newton's method for non-linear analysis was used with automatic stiffness update method. At the previous strain vs. time curves, strain curves of $\mathrm{FBG}_{\mathrm{B}}$ and $\mathrm{FBG}_{\mathrm{C}}$ definitely broke into two paths. Fig. 7 is load vs. strain curve for sensor line $\mathrm{FBG}_{\mathrm{B}}$. Fig. 7(a) is the exaggerated deformed shape of upper skin at $8 \mathrm{kN}$ load step. In the sensing point 1,4 and 3,6, the deformed shape by analysis shows out-of-plane-deflection in the upper direction of wing box, and in the sensing point 2,5 , the deformed shape by analysis shows out-of-plane-deflection in a downward direction of wing box. In the Fig. 7(b), (c), and (d), the slope of load-strain curves measured by FBG sensors show same tendency with analytic results. And the measured strain by FBG sensors shows good agreement with those by ESG. The path-breaking point of strain shows some differences between experiments and analysis. These differences may be caused by a defect of specimen, a disagreement of direction between loading and spars, etc.

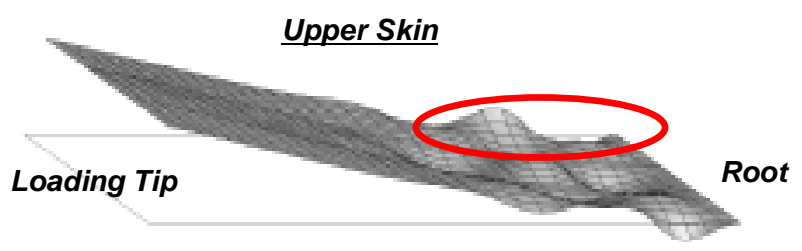

(a) Deformed shape of upper skin

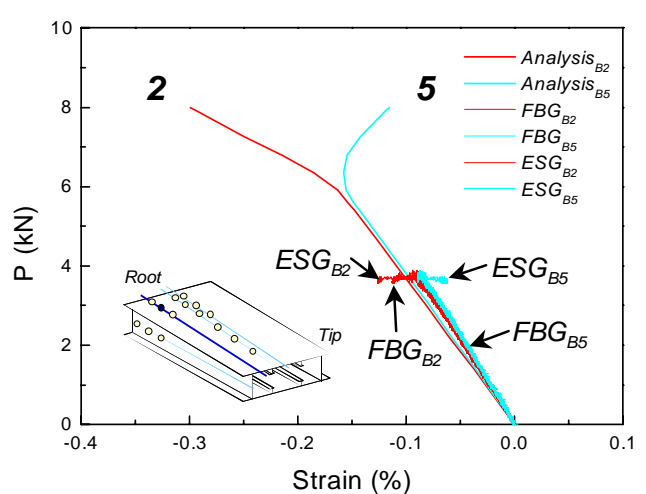

(c) Sensing point 2,5

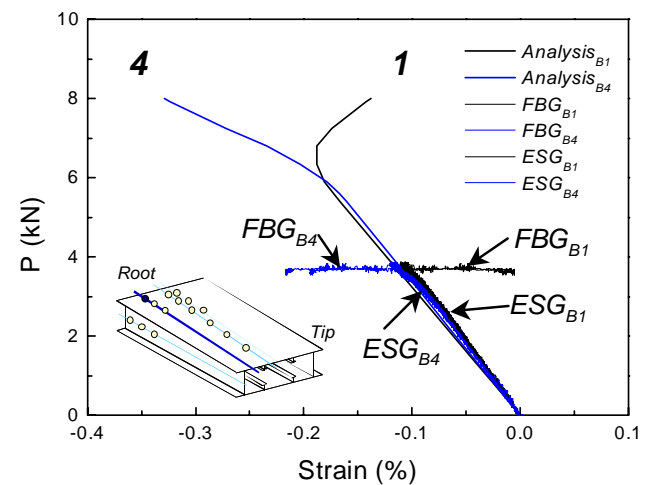

(b) Sensing point 1,4

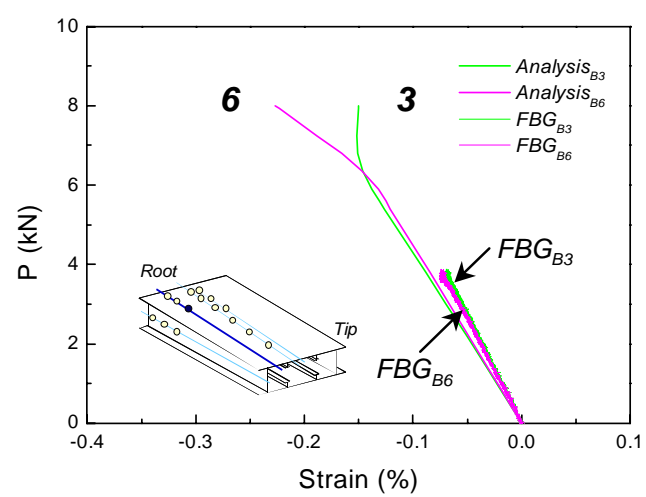

(d) Sensing point 3, 6

Fig. 7 Deformed shape of upper skin and load vs. strain curves by FBG $_{B}$ sensors during bending test.

Fig. 8 is load vs. strain curve for sensor line $\mathrm{FBG}_{\mathrm{C}}$. Fig. 8(a) is the exaggerated deformed shape of spars at $8 \mathrm{kN}$ load step. In the sensing point 1, 4 and 3,6, the deformed shape by analysis shows out-of-plane-deflection to the inside of wing box, and in the sensing point 2,5 , the deformed shape by analysis shows out-of-plane-deflection to the outside of wing box. In the Fig. 8(b), (c), and (d), the slope of load-strain curves measured by FBG sensors didn't show exactly same tendency with analytic results. But the measured strain by FBG sensors shows good agreement with those by ESG. Since the spars structurally sustain the most of bending load, the experimental buckling mode is very sensitive to any disturbance. These causes may make a difference between experiment and analysis. The $\mathrm{FBG}_{\mathrm{C}}$ sensor line was embedded into the mid-plane of the spar, which was also mid-plane of the wing box structure, so strain was relatively small compared to the strains from the sensor line A, B, D. The buckling behavior deepening phenomena were observed at the sensing point 1, 4 as shown in Fig. 8 (b). The buckling behavior of spars shown in Fig. 8 was in some different way compared to that of skin shown in Fig. 7 . The deepening phenomena of buckling behavior were not progressed from the root to loading tip of wing box in the test. Since the geometric shape of spar was tapered from the root to tip of wing box as shown in Fig. 4, the root part of spar sustain large bending load. The global buckling behavior occurred and progressed at the initial stage of the test in the 1, 4 sensing point. The experimental unsymmetrical and unstable mode of spar due to initial occurrence of global buckling mode made different results with the analysis which keeps symmetric mode. 


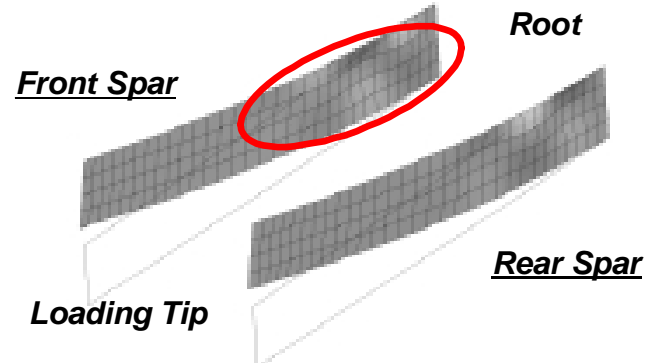

(a) Deformed shape of spars

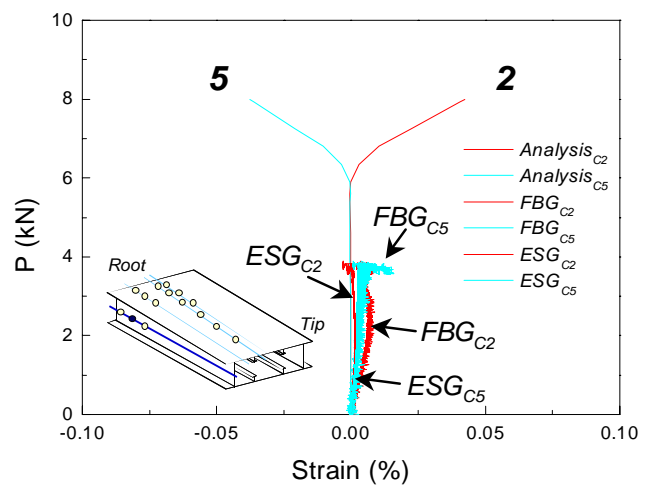

(c) Sensing point 2, 5

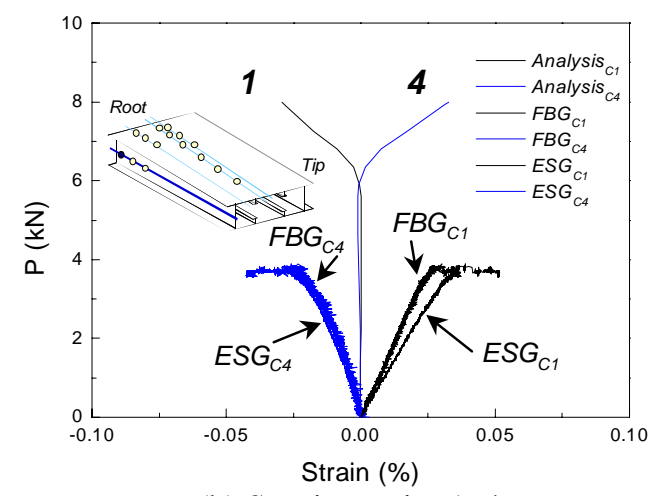

(b) Sensing point 1,4

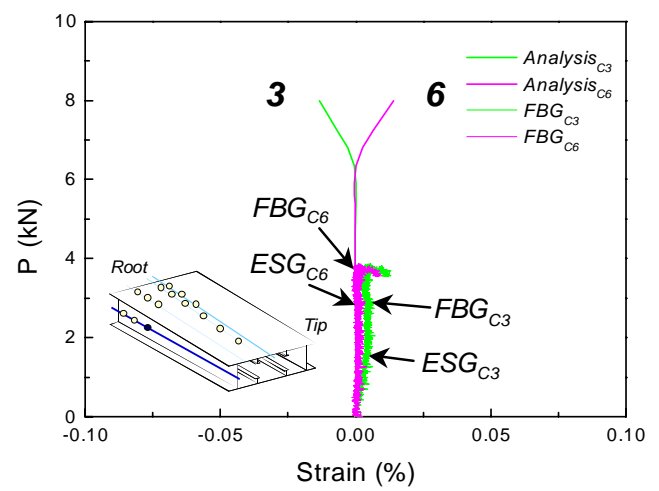

(d) Sensing point 3,6

Fig. 8 Deformed shape of spars and load vs. strain curves by $\mathrm{FBG}_{\mathrm{C}}$ sensors during bending test.

\section{CONCLUSIONS}

The behavior of composite wing box embedded 24 FBG sensors was real-timely monitored in the bending test. For this purpose, an improved FBG sensor system was constructed using the WSFL and a signal-processing board with an electrical circuit. It is possible to expand the number of sensor arrays using additional couplers and detectors by high power of the WSFL and digitalized signal-processing scheme. Four sensor lines where each sensor line has 6 sensors and 1 reference FBG were embedded into the skin and spar of composite wing box. The measured strain by FBG sensors showed good agreement with those by ESG. And experimental results showed same tendency with analytic results. The buckling behavior of upper skin and front spar of composite wing box was successfully detected and monitored by embedded FBG sensors. It was successfully demonstrated that the constructed FBG sensor system could be useful for the large structures that require a large number of sensor arrays.

\section{ACKNOWLEDGEMENTS}

The authors would like to thank the Ministry of Science and Technology, Korea, for the financial support by a grant from the Critical Technology 21 project.

\section{REFERENCES}

1. E. Udd, "Fiber Optic Smart Structures," Proceedings of the IEEE, 84, No. 1, pp. 60-67, 1996.

2. I. B. Kwon, C. G. Kim, and C. S. Hong, "Simultaneous Sensing of the Strain and Failure Instants of Composite Beams Using Fiber Optic Michelson Sensor,” Composites Science and Technology, 57, pp. 1639-1651, 1997. 
3. J. W. Park, and C. S. Hong, "Signal Characteristics in the Delaminated Composite Specimen with Fiber Optic Sensor," Proceedings of the 5th Japan International SAMPE Symposium, pp. 893-898, Tokyo, Japan, 1997.

4. J. W. Park, C. Y. Ryu, H, K, Kang, and C. S. Hong, "Detection of Buckling and Crack Growth in the Delaminated Composites Using Fiber Optic Sensor," Journal of Composite Materials, 34, No. 19, pp.1602-1623, 2000.

5. A. D. Kersey, T. A. Berkoff, and W. W. Morey, "Multiplexed Fiber Bragg Grating Strain-Sensor System with a Fiber Fabry-Perot Wavelength Filter," Optics Letters, 18, pp. 1370-1372, 1993.

6. Y. J. Rao, D. A. Jackson, L. Zhang, and I. Bennion, "Strain Sensing of Modern Composite Materials with a Spatial/ Wavelength-Division Multiplexed Fiber Grating Network," Optics Letters, 21, No. 9, pp. 683-685, 1996.

7. S. H. Yun, D. J. Richardson, and B. Y. Kim, "Interrogation of Fiber Grating Sensor Arrays with a Wavelength-Swept Fiber Laser," Optics Letters, 23, No. 11, pp. 843-845, 1998.

8. C. Y. Ryu, and C. S. Hong, "Development of Fiber Bragg Grating Sensor System Using Wavelength-Swept Fiber Laser," Smart Materials and Structures, submitted, 2000.

9. A. D. Kersey, M. A. Davis, T. A. Berkoff, D. G. Bellemore, K. P. Koo and R. T. Jones, "Progress Towards the Development of Practical Fiber Bragg Grating Instrumentation Systems," Proceedings of SPIE, 2839, 1997.

10. C. Y. Ryu, C. S. Hong, C. G. Kim, S. B. Lee, and S. S. Choi, "Stain Measurement of the Laminated Composites Using Attached Fiber Bragg Grating Sensor," Proceedings of the 3rd Optoelectronics and Communications Conference, pp. 272-273, Japan, 1998. 